\title{
Large Hamartomatous Polyp Presenting with Profuse Rectal Bleeding in Colo-colic Intussuception in A Child: A Case Report
}

\author{
K N Rattan ${ }^{1}$ Shruti Bansal2*, Roomi Yadav ${ }^{2}$, Gurupriya $\mathrm{J}^{2}$ and Neha Singh ${ }^{2}$ \\ ${ }^{1}$ Department of Paediatric Surgery, Pt. B.D. Sharma PGIMS Rohtak, Haryana, India \\ ${ }^{2}$ Department of Pathology, Pt. B.D. Sharma PGIMS Rohtak, Haryana, India
}

\begin{abstract}
Isolated colocolic intussusception in paediatric age group is quite rare with juvenile polyps being the most important pathological lead points. We are reporting a case of colocolic intussusceptionsecondary to solitary hamartomatous polyp as lead point in a 3.5 year oldmalechild who presented with profuse bleeding per rectum. The patient was successfully managed by reduction and colonic resection withcolo colic anastomosis and is doing well in follow up.
\end{abstract}

Keywords: Colocolic, Intussuception, Children, Solitary Hamartomatous Polyp

\section{Introduction}

Intussusceptionis a frequent cause of paediatric intestinal obstruction, seen most commonly in the childrenunder2 years of age with the peak incidence being in between 5 and 10 months of age. Colocolic intussusception (CCI) is an uncommon type of intussusception occurring in paediatric population with majority cases usually associated with a pathologic lead point such as juvenile polyps or tumor mass. ${ }^{[1]}$ To our knowledge, approximately 14 cases of CCI in children have been reported in literature till date with $65 \%$ of them associated with juvenile polyps as leading point for this variety of intussusceptions in the pediatric age group. We are reporting a case of colocolic intussusception with solitary hamartomatous polyp as lead point in a three and a half year old male child who presented with profuse rectal bleeding, highlighting the unusual presentation of a rare benign etiology of intussusception at an uncommon site in the child.

\section{Case Report}

A $12 \mathrm{~kg} 3.5$ year-old male child was brought to accident and emergency department by his parents with complaints of abdominal pain, profuse rectal bleeding and decreased acceptance. His past medical history was unremarkable. However, the patient was operated outside with the diagnosis of intussusception but it was negative laparotomy as surgeons failed to find intussusception. On physical examination, the child was conscious, irritable, afebrile and severely dehydrated with $\mathrm{BP}=100 / 60 \mathrm{mmHg}$, $P R=120 / \mathrm{min}, \mathrm{RR}=24 / \mathrm{min}$ and normal breathing. Growth and development was normal. Abdominal examination revealed soft and distended abdomen with no palpable lump. There was diffuse abdominal tenderness. Per rectal examination was negative. Chest was bilaterally clear and CVS examination was normal. All laboratory investigations including complete haemogram, coagulation studies, renal function test and serum electrolytes were within normal limits. The child was kept NPO and nasogastric aspiration done. Intravenous fluids and antibiotics were given and dehydration was corrected.

The child was investigated. X-ray abdomen was normal with no visualization of air fluid levels. On ultrasound, minimal intergut fluid was seen and there was telescoping of one gut loop into another at left hypochondrium and left lumbar region consistent with diagnosis of colo colic intussusception. After adequate resuscitation, child was taken up for surgery. Abdomen was opened through right supraumblical transverse incision. On exploration, colo colic intusucception was seen near the splenic flexure. It was reduced. After reduction, on palpation of large intestine, a firm intramural mass of $3.5 \times 3.5 \mathrm{cms}$ was palpable. On opening transverse colon, there was large polypoidal growth seen (Figure 1). Hence, resection and colo colic anastomosis was done. Abdomen was closed in layers. Postoperative period was uneventful with no recognizable bleeding. The child was started orally after 3 days of surgery and was discharged on $10^{\text {th }}$ post operative day. The patient recovered well from surgery and had no further episodes of intussusception. Colonoscopy was performed after 3 months to evaluate for other polypoid lesions but no polyps were found.

The resected colonic polyp was sent for histopathological examination. Pathologic examination revealed it to be solitary hamartomatous polyp without atypia (Figure 2\&3). 

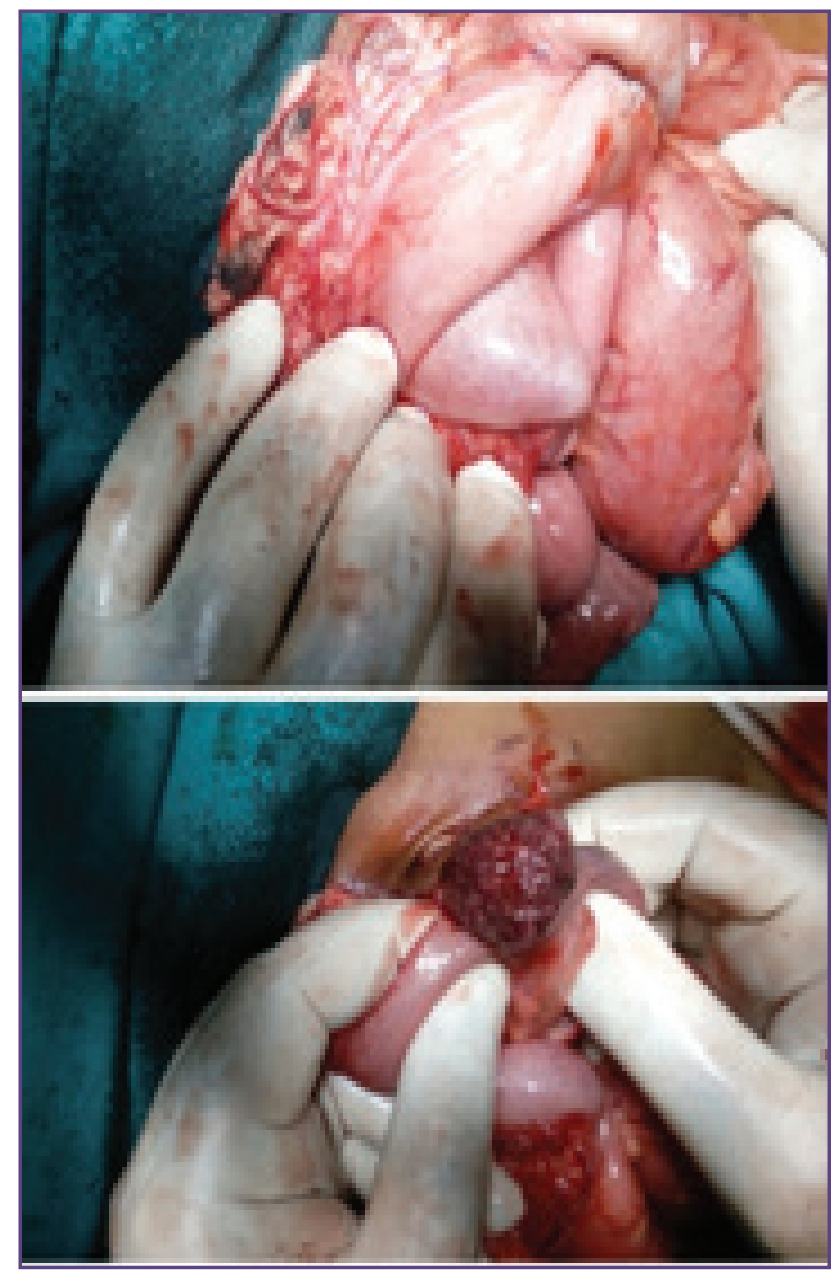

Fig. 1: Peroperative picture showing colo colic intussusception and solitary polyp.

\section{Discussion}

Intussusception accounts for up to $25 \%$ of abdominal emergencies in children up to the age of 5 years. The male-to-female ratio is approximately 3:2.The bowel may simply 'telescope' on itself (non-pathological lead point), or some pathology may be the focus of the invagination (pathological lead point).In approximately $95 \%$ of children, the intussusception is ileocaecocolic in location with about $90 \%$ cases being idiopathic in which no pathologic lead point is evident on barium enema or laparotomy. ${ }^{[2-3]}$ Hyperplasia of paeyers patches is the commonest cause for intussusception in children. Other types of intussusceptions namely jejunojejunal or colocolic are quite rare, usually have a pathological lead point and are seen more commonly in children older than 2 years of age. Possible lead points include intestinal polyps, Meckels diverticulum, Peutz-Jeghers syndrome, mucosal hematoma, Henoch-Schoenlein purpura, neoplasms, lipoma and intestinal duplications.

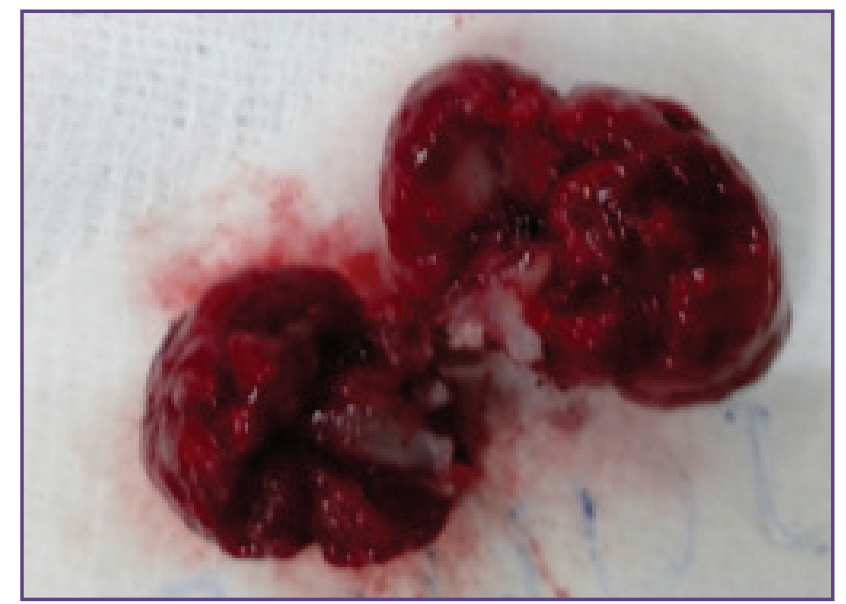

Fig. 2: Resected polypectomy specimen.

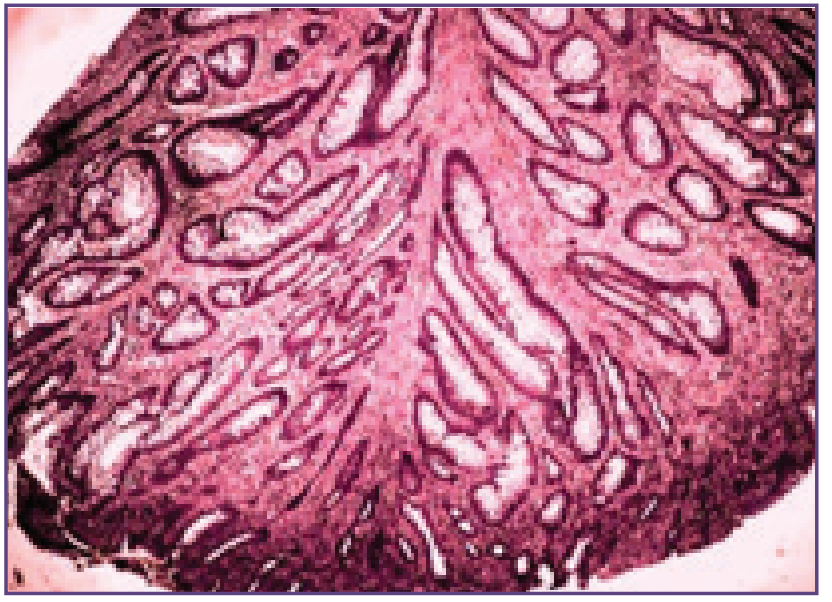

Fig. 3: Photomicrograph showing histopathology of solitary hamartomatous polyp.

Colo-colic intussusception is an unusual cause of large bowel obstruction in children with no studies documenting the exact prevalence of the disease. Its occurrence is mostly associated with a pathological lead point with majority case reports reporting it to be juvenile polyps. However, the polyps varied in size and clinical presentation. Arthur et al reported a case of colocolic intussusception in a three year old child caused by a colonic polyp. ${ }^{[3]} \mathrm{A}$ case of CCI in a four year old male secondary to juvenile polyps was reported by Abrahamsetal in 2012. The child came with complaints of worsening vomiting and diarrhea and on exploration was found to have two large intraluminal pedunculated polyps (measuring $3.3 \times 2.5 \times 2.0 \mathrm{~cm}$ and $2.2 \times 2.0 \times 1.0 \mathrm{~cm}$ ) and one small polyp as lead points for CCI. ${ }^{[4]}$ Yamada et al reported CCI in 10 month old boy who presented with bloody stools. Juvenile polyp approximately $15 \mathrm{~mm}$ in size was found to be the leading point. ${ }^{[5]}$ However, two cases of CCI without pathologic lead points had been reported in a 7-year-old boy in 2008 by Mahmudloo et al and in 
a 4 year old boy by Sanchez et al. ${ }^{[6-7]}$ An unusual case of neonatal CCI with intestinal lymphangioma as lead point was reported by Al-Jahdali et al in 2009. ${ }^{[8]}$ Pediatric CCI caused by malignancy has been reported rarely in the literature. A rare case in a 5-year-old child who presented with intermittent CCI with sigmoid colon ganglioneuroma as leading point was reported by Soccorso et al. ${ }^{[9]}$ Although the leading point in our case was colonic polyp similar to that reported in significant number of cases, but its large size of $3.5 \times 3.5 \mathrm{cmsand}$ clinical presentation of profuse bleeding per rectum was quite distinct.

The clinical manifestations in majority of these cases are paroxysms of colicky abdominal pain and lower intestinal bleeding. Abdominal radiography is the most common initial study used in the workup of abdominal pain and suspected intussusception. Abdominal X-rays may show dilated gas-filled proximal bowel with paucity of gas distally and multiple air fluid levels but they generally have low sensitivity and specificity for the diagnosis of intussusception as a quarter of intussusception cases have a normal abdominal radiograph. ${ }^{[10]}$

The well established imaging modality for the diagnosis of intussusception is an abdominal ultrasonography. It is quite sensitive, specific, reliable and inexpensive tool for diagnosing intussusception and can also help in identification of pathological lead points. Sonographically, the most frequently described appearance of intussusception is a bull's eye or target-like lesion on transverse views which is formed by a loop of bowel (intussusceptum) within another loop of bowel (intussuscepient). Also, the affected bowel segment on longitudinal view resembles the appearance of a kidney which is referred to as pseudo-kidney. CT scan reveals the presence of a target or sausage-shaped lesion and also has high diagnostic accuracy rate. Nevertheless, colonoscopy is a highly useful tool for evaluating patients presenting with intussusception.

\section{Conclusion}

Any paediatric patient presenting with fresh rectal bleeding with evidence of intussusception on ultrasonography should be subjected to colonoscopy for confirmation of polypoidal lesions. Colonoscopy, under appropriate sedation, is beneficial for both diagnostic and curative purposes. Colonoscopic polypectomy performed by an experienced endoscopist, is generally more useful than open surgical treatment when organic lesions such as juvenile polyps are present.

\section{References}

1. Das A, Ralte L, Chawla AS, Arya SV, Kumar A, Saroha R et al. Colocolic Intussusception in an Older Child: A Rare Case Report and a Literature Review. Case Rep Surg. 2013;2013:1-3.

2. Dominique TLM. Lower gastro-intestinal bleeding. In: Allan Walker W, Kleinman RE, Sanderson IR, et al (eds). Pediatric Gastrointestinal Disease. 4th ed. Hamilton, Ontario; BC Decker. 2004; p273.

3. Arthur AL, Garvey R, Vaness DG. Colocolic intussusception in a three-year-old child caused by a colonic polyp. Conn Med. 1990;54:492-4.

4. Abrahams RB, Franco A, Lewis KN. Pediatric colocolic intussusception with pathologic lead point: a case report. J Med Cases. 2012;3:84-8.

5. Yamada S, Miura T, Nakamura J, Emura I. Pediatric colocolic intussusception due to juvenile polyp. Gastroenterological Endoscopy. 2010;52:1267-74.

6. Mahmudloo R, Gheibi S, Vahed SN. Colocolic Intussusception without Lead Point; A Case Report and Literature Review. Iran J Pediatr. 2008;18:373-6.

7. Sanchez S, Javid P, Ricca R, Avansino J. Colocolonic intussusception in a four-yr-old with a heart transplant: A case report and review of the literature. Pediatr Transplant. 2011.

8. Al-Jahdali A, Lees GM, Gay DP, Al-Sairafi R. Colocolic intussusception in a preterm infant with intestinal malrotation. J Pediatr Surg. 2009;44:e17-18.

9. Soccorso G, Puls F, Richards C, Pringle H, Nour S. Aganglioneuroma of the sigmoid colon presenting as leading point of intussusception in a child: a case report. JPediatrSurg. 2009;44;e17-e20.

10. Hernandez JA, Swischuk LE, Angel CA. Validity of plain films in intussusception. EmergRadiol. 2004;10:323-6.

*Corresponding author:

Dr. Shruti Bansal, Department of Pathology, Pt. B.D. Sharma PGIMS, Rohtak, Haryana, India

Phone: +9107206341546

Email: shruti.b.bansal3@gmail.com

Financial or other Competing Interests: None.

Date of Submission : 11.08.2016

Date of Acceptance : 08.01.2017

Date of Publication : 26.03.2017 